\title{
First-principles coupled cluster theory of the electronic spectrum of transition metal dichalcogenides
}

\author{
Artem Pulkin* and Garnet Kin-Lic Chan \\ Division of Chemistry and Chemical Engineering, California Institute of Technology, Pasadena, California 91125, USA
}

(Received 7 October 2019; revised manuscript received 22 May 2020; accepted 26 May 2020; published 26 June 2020)

\begin{abstract}
The electronic properties of two-dimensional transition metal dichalcogenides (2D TMDs) have attracted much attention during the last decade. We show how a diagrammatic ab initio coupled cluster singles and doubles (CCSD) treatment paired with a careful thermodynamic limit extrapolation in two dimensions can be used to obtain converged band gaps for monolayer materials in the $\mathrm{MoS}_{2}$ family. We find CCSD gaps to lie in the upper range of the spread of $G W$ approximation based on density functional theory (DFT) simulations, and also find slightly higher effective hole masses compared to previous reports. We also investigate the ability of CCSD to describe trion states, finding a reasonable qualitative structure, but poor excitation energies due to the lack of screening of three-particle excitations in the effective Hamiltonian. Our study provides an independent high-level benchmark of the role of many-body effects in 2D TMDs and showcases the potential strengths and weaknesses of diagrammatic coupled cluster approaches for realistic materials.
\end{abstract}

DOI: 10.1103/PhysRevB.101.241113

Two-dimensional (2D) materials are flat, atomically thin crystals [1-3] that can be prepared through a number of wellestablished techniques [4-6]. They display diverse electronic structures ranging from conventional semiconductors with an optical band gap [7] to topological insulators [8,9], materials hosting unconventional magnetism [10,11], superconductivity [12,13], and spin physics [14,15]. 2D transition metal dichalcogenides (TMDs) $M X_{2}, M=\mathrm{Mo}, \mathrm{W}, \mathrm{Nb}, \mathrm{Ti}, \ldots, X=$ $\mathrm{S}, \mathrm{Se}, \mathrm{Te}$ are among the most promising representatives of this family, with potential applications in electronics [4,16-20] and beyond $[9,12,14]$.

The electronic properties of the $2 \mathrm{H}$ structural phase of monolayer TMDs and $2 \mathrm{H}-\mathrm{MoS}_{2}$ in particular have been studied extensively through spectroscopy [18,21-24], and transport $[17,18]$ experiments. Several studies have probed the electronic band structure directly $[4,7,21,25-30]$. A large number of theoretical works based on model or first-principles Hamiltonians [the latter in conjunction with various flavors of density functional theory (DFT) and the DFT- $G W$ approximation] have been carried out to complement the experimental picture [31-47]. Although broad agreement between theory and experiment is observed, there is uncertainty at the quantitative level in numerical results arising from different methodologies, with estimates of the band gap varying over a range of $0.4 \mathrm{eV}$ or more even from different flavors of DFT$G W$ [43]. In this context, independent high-level many-body benchmarks of the electronic properties of monolayer TMDs remain desirable.

*Present address: QuTech and Kavli Institute of Nanoscience, Delft University of Technology, 2600 GA Delft, The Netherlands; gpulkin@gmail.com

${ }^{\dagger}$ gkc1000@gmail.com
The $G W$ approximation has long been the state of the art in the $a b$ initio many-body description of materials singleparticle spectra. More recently, however, coupled cluster (CC) theory, widely used in accurate molecular calculations, and in particular, the equation-of-motion coupled cluster (EOMCC) theory, the extension to excited states and spectra, has emerged as a viable approach for extended systems [48-53]. In molecular systems, benchmarks find that EOM-CC at the singles and doubles level of approximation (EOM-CCSD) gives a large reduction in errors relative to the $G W$ approximation [54] (e.g., by a factor of 4-8 in the ionization potential depending on the variant of $G W$ ). More limited studies have also demonstrated the superiority of EOM-CC in materials, in particular in more correlated systems [53,55]. From a theoretical perspective, the diagrammatic content of $\mathrm{CC}$ theory means that it has a greatly reduced dependence on the mean-field starting point [55], while a careful diagrammatic analysis of EOM-CCSD shows that it includes large classes of diagrams that are omitted in the standard $G W$ approximation [54].

In the present Rapid Communication we report benchmark single-particle band gaps for the monolayer TMDs and the full band structure for the representative monolayer material $2 \mathrm{H}-\mathrm{MoS}_{2}$, obtained from first-principles EOM-CCSD calculations. We discuss the role of dimensionality in finite-size effects which are the largest source of uncertainty in our results. The EOM-CC band gaps and the monolayer $2 \mathrm{H}-\mathrm{MoS}_{2}$ band structure are found to be quite close to previous $G W$ results, demonstrating that additional many-body effects beyond those in EOM-CCSD are likely very small. We analyze the remaining uncertainties in our computed band structure arising from spin-orbit coupling. Finally, we examine the three-particle excited states of the coupled cluster Hamiltonian and compare them to trions observed in these materials. 
We describe the 2D material in terms of an all-electron Hamiltonian with periodic boundary conditions (PBCs) expressed in a finite crystalline molecular orbital (Bloch) basis,

$$
H=\sum_{\alpha \beta k} h_{\alpha \beta k} \mathbf{c}_{\alpha k}^{\dagger} \mathbf{c}_{\beta k}+\sum_{\substack{\alpha \beta \gamma \delta \\ k_{1} k_{2} k_{3}}} v_{\substack{\left(\alpha k_{1}\right),\left(\beta k_{2}\right) \\\left(\gamma k_{3}\right),\left(\delta k_{4}\right)}} \mathbf{c}_{\alpha k_{1}}^{\dagger} \mathbf{c}_{\beta k_{2}}^{\dagger} \mathbf{c}_{\delta k_{4}} \mathbf{c}_{\gamma k_{3}},
$$

where $\mathbf{c}_{\alpha k}$ is a fermionic annihilation operator removing one electron from the crystalline molecular spin-orbital $\alpha k(\alpha$ and other Greek symbols denote orbitals, $k$ is the Bloch wave vector), $h$ denotes single-particle Hamiltonian matrix elements corresponding to the sum of kinetic and external potential energies of the electrons, and $v$ denotes the Coulomb repulsion matrix element for the four crystalline molecular orbitals $\left(k_{4}\right.$ satisfies pseudomomentum conservation in the scattering process: $\left.k_{1}+k_{2}=k_{3}+k_{4}\right)$. The crystalline molecular orbitals $\psi_{\alpha k}(r)$ are expanded in terms of crystalline Gaussian atomic orbitals $\tilde{\phi}_{\lambda k}$, i.e., $\psi_{\alpha k}=\sum_{\lambda} u_{\alpha \lambda} \tilde{\phi}_{\lambda k}$, with $\tilde{\phi}_{\lambda k}(r)=\sum_{R} \phi_{\lambda}(r-R) e^{i k \cdot R}$, where $\phi_{\lambda}(r)$ is a Gaussian basis function centered at $r=0 ; R$ are lattice vectors. In this work we used the double-zeta valence polarized (DZVP) basis set [56] for dense $k$-point calculations of monolayer $2 \mathrm{H}-\mathrm{MoS}_{2}, 2 \mathrm{H}-\mathrm{MoSe}_{2}$ and the triple-zeta valence polarized (def2-TZVP) [57] basis set was used for the tungsten materials; lattice parameters were taken from Ref. [32]. The wave vectors $k$ were chosen to sample the Brillouin zone (BZ) uniformly. The Coulomb integrals $v$ can formally be defined to be finite in reciprocal space,

$$
\begin{aligned}
v_{\left(\alpha k_{1}\right),\left(\beta k_{2}\right)} & =v_{1234}=\int d G \cdot K(G) \rho_{13}(G) \rho_{24}(-G) \\
\left(\gamma k_{3}\right),\left(\delta k_{4}\right) & \\
& \approx \sum_{G \neq 0} w \cdot K(G) \rho_{13}(G) \rho_{24}(-G)
\end{aligned}
$$

where $G$ are reciprocal lattice vectors, $K(G)=\int d r \cdot e^{-i G \cdot r} / r$ is the Coulomb kernel; $\rho_{i j}(G)=\int d r e^{-i G \cdot r} \psi_{i}^{*}(r) \psi_{j}(r)$ are Fourier transforms of pair densities ( $r$ belongs to the unit cell); $w$ is the volume of the reciprocal-space grid box. In this work, we used Gaussian density fitting [58,59] to compute $v$ efficiently.

We determined the spectrum by successively finding ground-state spin-restricted Hartree-Fock (HF), ground-state CCSD, and excited-state EOM-CCSD solutions in the $N_{e}+1$ (electron affinity, EA) and $N_{e}-1$ (ionization potential, IP) sectors $\left[N_{e}\right.$ is the number of electrons per unit cell multiplied by the number of $k$ points sampled in the Brillouin zone (BZ)]. An "active" single-particle space for the correlated calculations was restricted to the seven hole and seven electron bands adjacent to the band gap. The choice of seven valence bands takes into account all valence energy shells of Mo and S elements, other than the $3 s$ band of the sulfur atoms: The corresponding deep valence bands are isolated by a $\sim 10-\mathrm{eV}$ gap from the rest of the valence states. Seven conduction bands within an 8-eV energy window then form the remainder of this active space. In Fig. 1(d) we show the error in the resulting band gap size from using this active space relative to the full basis for some smaller $k$ meshes; the
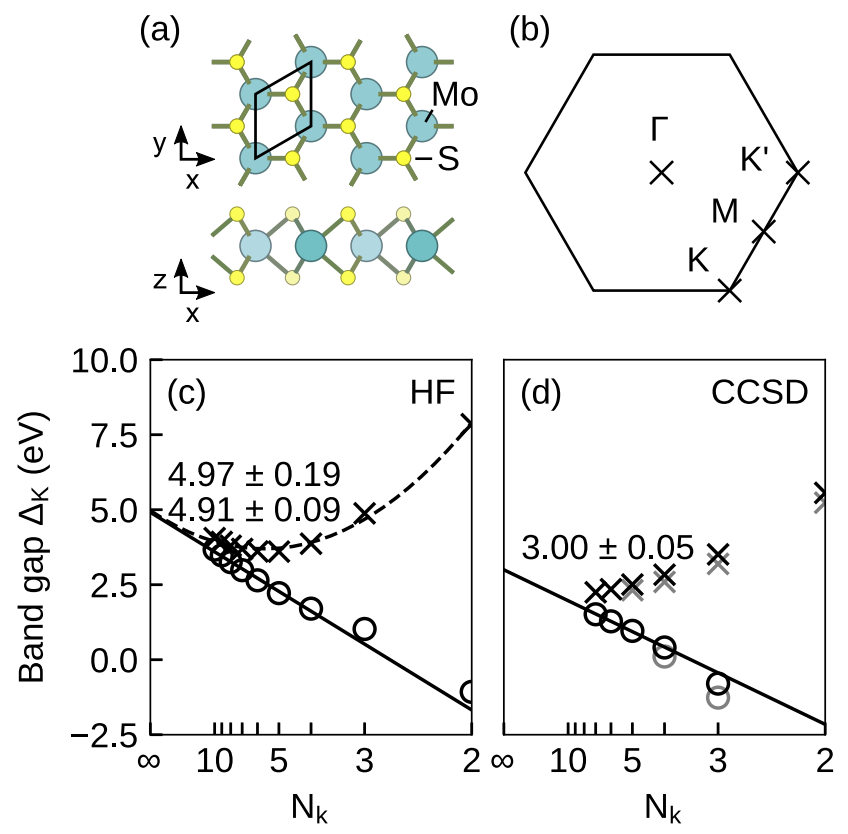

FIG. 1. Atomic structure and the size of the computed band gap in monolayer $2 \mathrm{H}-\mathrm{MoS}_{2}$. (a) Atomic structure of monolayer $2 \mathrm{H}-\mathrm{MoS}_{2}$ : top and side views. (b) High-symmetry points $\Gamma, M, K$, $K^{\prime}$ in the hexagonal Brillouin zone. (c), (d) Extrapolation of HF and CCSD band gaps at the high-symmetry $K$ point $\Delta_{K}$. Solid lines and circles are obtained from the 2D approach when calculating the band gap; the dashed line and crosses correspond to the $3 \mathrm{D}$ approach (see main text). The CCSD band gaps calculated using the full DZVP basis set are presented as a reference (gray symbols). The number of $k$ points along one reciprocal axis $N_{k}$ (horizontal axis) is presented on the $N_{k}^{-1}$ scale. The extrapolated $N_{k} \rightarrow \infty$ band gap sizes and the corresponding standard deviations are indicated. Negative values of the band gap reflect the overlap of occupied and virtual single-particle spaces due to the finite-size error discussed in the main text.

error is much smaller than other uncertainties in this work. All calculations were performed using the PYSCF package [58], and other simulation details (basis sets, cutoffs) are given in the Supplemental Material [60].

Apart from the intrinsic many-body error associated with the CCSD approximation and from the Gaussian basis, a dominant source of uncertainty comes from the finite-size effects due to finite sampling of the BZ [61-67]. This is most evident in the Coulomb matrix elements due to the slowly decaying tail of the Coulomb operator for $r \rightarrow \infty, G=0$. In Hartree-Fock theory one often corrects for this finite-size error by recognizing that the $G=0$ singularity in the Coulomb operator can be canceled by the exchange density matrices to obtain a finite value, leading to a variety of finite-size correction schemes [68]. However, it is less clear how to consistently apply such schemes in the context of a correlated coupled cluster calculation. Consequently, we perform both our Hartree-Fock and coupled cluster calculations without such finite-size corrections, and instead correct the $G=0$ error by extrapolating observable values. For example, we performed several band structure calculations with different samplings (numbers of $k$ points $N_{k}^{2} \leqslant 49$ ) of the $2 \mathrm{D} \mathrm{BZ}$ and 
extrapolated the band gap size with a known law (linear or quadratic polynomials in Fig. 1). The corresponding uniform $k$-point grids were shifted to always include the $K$ point explicitly.

The calculated values of $\Delta_{K}$ for monolayer $2 \mathrm{H}-\mathrm{MoS}_{2}$ are presented in Fig. 1 for both HF (the single-particle energy difference) and CCSD (the difference between EA and IP eigenvalues) as a function of the number of $k$ points along one reciprocal axis $N_{k}$. Two data sets are presented in each case, "2D" and "3D," standing for two possible approaches to calculating matrix elements $h$ and $v$. The "3D" approach is the conventional way to describe a 2D crystal with PBCs in three dimensions: Periodic images of a 2D material are separated with a large but finite vacuum in the $z$ direction while the integral Eq. (2) is carried out in three dimensions with a 3D Coulomb kernel $K=4 \pi / G^{2}$. The "2D" approach assumes PBCs in two dimensions only ( $x y$ plane) while the $z$ direction is under "infinite" boundary conditions and is integrated out in real space. The Coulomb kernel in this case can be found, e.g., in Ref. [68] and in the Supplemental Material [60].

The two approaches are equivalent only in the $N_{k} \rightarrow \infty$ limit while finite-size errors behave differently in each case. The finite-size error in the $2 \mathrm{D}$ case can be estimated as the magnitude of the first nonzero term in Eq. (2),

$$
\operatorname{err}_{2 \mathrm{D}}(\Delta) \sim w_{2} / G_{\min } \sim 1 / N_{k},
$$

where $G_{\min }$ is the spacing of the 2D reciprocal grid and $w_{2}$ is the surface area of the reciprocal $2 \mathrm{D}$ grid. The same approach in $3 \mathrm{D}$ yields a different error estimate,

$$
\operatorname{err}_{3 \mathrm{D}}(\Delta) \sim w_{3} / G_{\min }^{2} \sim \max \left(N_{k}, z\right)^{2} /\left(z N_{k}^{2}\right),
$$

where $w_{3}$ is the volume of the reciprocal 3D box and $z$ stands for the ratio between the vacuum size and the lattice constant: $z \sim 20 \AA / 3.193 \AA \approx 6.26$ in the monolayer $2 \mathrm{H}$ - $\mathrm{MoS}_{2}$ model presented. Equation (4) takes two possible limits: $\operatorname{err}_{3 \mathrm{D}}(\Delta) \sim z / N_{k}^{2}, z \gg N_{k}$ and $\operatorname{err}_{3 \mathrm{D}}(\Delta) \sim 1 / z, z \ll N_{k}$. This is consistent with the behavior presented in Fig. 1: While the $2 \mathrm{D}$ treatment results in a clear $1 / N_{k}$ trend for both the Hartree-Fock and CCSD band gap, $\Delta\left(N_{k}\right)$ calculated with the $3 \mathrm{D}$ treatment exhibits a minimum corresponding to the transition at $N_{k} \sim z$. Because of the high computational cost of the CCSD calculations, we were restricted to a maximum $N_{k, \text { max }}=7$, comparable to the vacuum size $z$, prohibiting a meaningful extrapolation of the CCSD band gap in the 3D case. In particular, it is clear that the 3D CCSD data have yet to reach the linear extrapolation regime. However, in the case of the HF gap, where we computed up to a larger $N_{k \text {, max }}=$ 10, the two approaches agree for the extrapolated HF band gap size [Fig. 1(c)]. Given the greater reliability of the 2D extrapolation for CCSD with the $k$-point meshes we could sample in this work, we will henceforth only discuss results from the $2 \mathrm{D}$ treatment.

Previous studies have found a range of values for the monolayer $2 \mathrm{H}-\mathrm{MoS}_{2}$ fundamental band gap: $1.9-2.2 \mathrm{eV}$ [scanning tunneling spectroscopy (STS) experiments [29,30]], $1.7-1.8 \mathrm{eV}$ (DFT calculations [31,32,36]), 1.8-2.3 eV (hybrid functional DFT calculations [33,41,69]), 2.6-3.0 eV (DFT-based $G W$ calculations [34,39,42-44]), while the optical band gap ranges from 1.8 to $1.9 \mathrm{eV}$ (optical spectroscopy $[4,7,21,25])$, and 1.8 to $2.2 \mathrm{eV}$ [ $G W$ Bethe-Salpeter
TABLE I. Band gap sizes across four monolayer TMDs in eV. The value in parentheses indicates an indirect $\Gamma-K$ band gap. The last row is calculated as $\Delta_{\mathrm{CCSD}^{*}}=\Delta_{\mathrm{CCSD}}-\mathrm{SOC} / 2$, where SOC are spin-orbit-coupling-induced valence band splittings taken from Ref. [38]. * Other reference $G W$ data include fundamental band gap values from Refs. [34,42,44,45] $\left(\mathrm{MoSe}_{2}\right)$, Refs. [42,44] $\left(\mathrm{WS}_{2}\right)$, and Refs. [42,44,46,47] $\left(\mathrm{WSe}_{2}\right)$, in addition to Ref. [38].

\begin{tabular}{lcccc}
\hline \hline & $\mathrm{MoS}_{2}$ & $\mathrm{MoSe}_{2}$ & $\mathrm{WS}_{2}$ & $\mathrm{WSe}_{2}$ \\
\hline$\Delta_{\mathrm{PBE}}[38]$ & 1.60 & 1.35 & 1.56 & 1.19 \\
$\Delta_{G W}[38]$ & 2.82 & 2.41 & 2.88 & 2.42 \\
$\Delta_{G W}\left({ }^{*}\right.$ other $)$ & $2.58-2.97$ & $2.18-2.60$ & $2.43-2.88$ & $2.08-2.82$ \\
$\Delta_{\mathrm{CCSD}}$ & $3.00(2.93)$ & 2.63 & 3.23 & 3.01 \\
$\Delta_{\mathrm{CCSD}^{*}}$ & 2.92 & 2.52 & 3.00 & 2.76 \\
\hline \hline
\end{tabular}

equation ( $G W$-BSE) calculations $[39,43]]$. We find an extrapolated CCSD band gap value of $3.00 \pm 0.05 \mathrm{eV}$. The error bar is the statistical uncertainty of the intercept as produced by the fit, although we note that it may be an underestimate due to the small number of data points. There is a spread of DFT$G W$ results over about $0.4 \mathrm{eV}$, and the CCSD value is close to (and slightly higher than) the upper end from Ref. [38]. Given the largest difference between the DFT- $G W$ and CCSD values is $0.4 \mathrm{eV}$, and the performance relative to exact gaps seen in molecular comparisons of the two methods [54], we expect the remaining many-body effects beyond those in the EOM-CCSD approximation to be smaller than this number, and thus (relatively) modest in size. However, we also see that the difference between the CCSD and DFT- $G W$ band gap size is significantly larger for other materials in the same family $\left(\mathrm{MoSe}_{2}, \mathrm{WS}_{2}, \mathrm{WSe}{ }_{2}\right.$, see Table I), with the largest differences associated with the heavier elements. This is likely related to the absence of spin-orbit splitting of the valence bands in our model. An estimate of the band gap size $\Delta_{K}$ for the spin-orbit splitting is presented in the last row of Table I, where the CCSD band gap value is combined with the $G W$ spin-orbit splitting size. The corrected band gap sizes of monolayer $2 \mathrm{H}-\mathrm{MoS}_{2}, 2 \mathrm{H}-\mathrm{MoSe}_{2}$, and $2 \mathrm{H}-\mathrm{WS}_{2}$ are then more similar to the $G W$ values in Ref. [38]. However, the monolayer $2 \mathrm{H}$-WSe $2 \mathrm{CCSD}$ band gap remains $340 \mathrm{meV}$ larger, suggesting additional correlation effects play a role in this material.

Most earlier theoretical and experimental studies of monolayer $2 \mathrm{H}-\mathrm{MoS}_{2}$ agree that the fundamental band gap is direct and located at the $K, K^{\prime}$ points of the hexagonal BZ. However, bilayer, multilayer, bulk $[18,35,37,70]$, as well as deformed monolayer crystals of $2 \mathrm{H}-\mathrm{MoS}_{2}[36,70-72]$ are also known to have an indirect band gap originating from the the maximum of the valence bands located at the $\Gamma$ point. In addition, some $G W$ studies [40,73] have suggested a different location of the conduction band minimum occurring along the $\Gamma-K$ high-symmetry path but not at the $K$ point itself. These observations are explained by a rather small $\sim 100 \mathrm{meV}$ difference between the corresponding band extrema [7]. To investigate this question in detail, we plot the EOM-CCSD electronic band structure of monolayer $2 \mathrm{H}-\mathrm{MoS}_{2}$ and compare it to other methods in Fig. 2. The band structure we obtain contains an indirect $\Gamma-K$ band gap $2.93 \mathrm{eV}$ as shown in Fig. 2. However, 


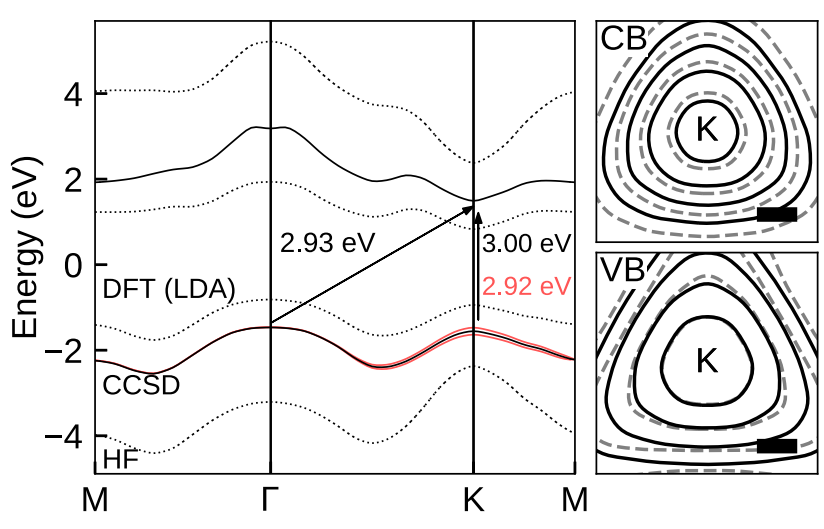

FIG. 2. Electronic band structure of monolayer $2 \mathrm{H}-\mathrm{MoS}_{2}$. Left: Electronic band structure of monolayer $2 \mathrm{H}-\mathrm{MoS}_{2}$ along the highsymmetry $M-\Gamma-K-M$ path: CCSD bands (solid lines), HF and LDA DFT bands (dashed lines), and the spin-orbit postprocessing correction to CCSD valence bands (red color). Arrows indicate CCSD direct and indirect band gaps. HF and CCSD bands are shifted to match the extrapolated band gap size. Right: Shapes of valence (VB) and conduction bands (CB) close to the $K$ point: CCSD (solid) and LDA DFT (dashed) lines. The isolines are plotted with a $100-\mathrm{meV}$ step starting from the corresponding band extrema at the $K$ point. The scale bar is $0.1 \AA^{-1}$.

the difference between the $\Gamma-K$ and $K-K$ transitions is $\sim 70$ meV: This is less than a typical spin-orbit-induced valence band shift $150 / 2=75 \mathrm{meV}$ [21]. Thus, a direct band gap $\Delta_{K}$ is expected upon the inclusion of the spin-orbit coupling into the model. Red bands in Fig. 2 demonstrate the spin-orbit splitting of $164 \mathrm{meV}$ [38] added to the CCSD bands as an illustration of the expected behavior. On the other hand, localdensity-approximation (LDA) DFT bands obtained within the same setup (structural parameters, basis set, no spin-orbit band splitting) and plotted in Fig. 2 also possess an indirect 1.61-eV band gap with a $110-\mathrm{meV}$ difference between valence band extrema at $K$ and $\Gamma$. In contrast, HF bands form a pronounced direct band gap at the $K$ point. Note that these observations are subject to the choice of lattice parameters: A relatively small strain is known to cause a pronounced indirect band gap in both DFT and $G W$ models of the material $[37,40]$ and the same effect is expected here.

To compare band dispersion features, we combined band values from several EOM-CCSD $6 \times 6$ uniformly shifted $k$-point calculations into a larger $18 \times 18 k$-point data set shown in Fig. 1. From this data we deduced $K$-point electron and hole masses $m_{e}^{*}=0.48 m_{e},\left|m_{h}^{*}\right|=0.75 m_{e}$. This result is in line with previous predictions [35,38,40,74-76] $m_{e}^{*}=$ $0.3-0.6 m_{e},\left|m_{h}^{*}\right|=0.35-0.65 m_{e}$, and experiments [28] $\left|m_{h}^{*}\right|=$ $0.43-0.48 m_{e}$. The larger deviation of the hole mass is partially explained by the aforementioned absence of the spinorbit splitting in the model, which would otherwise increase the energy scale and reduce the effective mass of the hole quasiparticles. To support this explanation, we calculated the effective hole mass from the LDA DFT band structure under the spin-restricted approximation and observed a similar effect: $\left|m_{h}^{*(\mathrm{DFT})}\right|=0.70 m_{e}$ [see Fig. 2 (side panels) for the comparison of the bands' profiles].
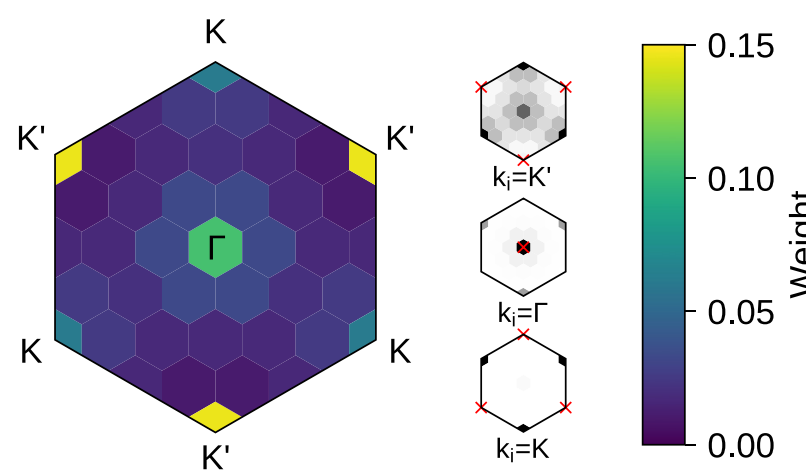

FIG. 3. Weights of the trion-like IP root (color) in the EOM CCSD spectrum of monolayer $2 H-\mathrm{MoS}_{2}\left(1 p 2 h\right.$ weight $w_{1 p 2 h}>$ 0.99). The left image shows weights of the trion in the hexagonal Brillouin zone $(6 \times 6$ sampling $) w\left(k_{i}\right)=\sum_{k_{j} ; i j a}\left|r_{i j a}^{(2)}\left(k_{i}, k_{j}\right)\right|^{2}$, where $r^{(2)}$ are the three-particle amplitudes of the solution. The three side images resolve $w\left(k_{j}\right)=\sum_{i j a} r_{i j a}^{(2)}\left(k_{i}, k_{j}\right)$ for the $k_{i}$ contributing the largest weights in the first plot.

Finally, we examine the EOM-CCSD multiparticle excitations. Trions in monolayer $2 \mathrm{H}-\mathrm{MoS}_{2}$ and similar materials have been observed in several experiments [25,77-81] where a large 10-meV-order binding energy between charged and charge-neutral excitations was measured. We focused on trion roots in our model that are closest to the Fermi level (the example shown in Fig. 3). All trions we observed couple single-particle states belonging to the topmost valence band and lowest-lying conduction band. The primary contributions to the EOM $r^{(2)}$ amplitudes originate from either the $K$ and $K^{\prime}$ points (both electrons and holes) or the $\Gamma$ point (holes only) which are closest to the Fermi level in our model, as expected. Figure 3 demonstrates a root coupling an exciton at the $K^{\prime}$ point to a hole at the $K$ point. There is also a significant contribution of a double-hole excitation at $\Gamma$ plus an electron at the $K^{\prime}$ point to this root. The trion solution shown in Fig. 3 is nearly degenerate with at least five other similar roots with a total momentum $k=K, K^{\prime}$ pointing to the existence of dark excitons in the material [81]. However, the calculated energies of the trions roughly correspond to those in the Hartree-Fock single-particle picture $\epsilon_{i}+\epsilon_{j}-\epsilon_{a}$. This results from the inability of EOM-CCSD to describe screening in three-particle excitations. To understand this, note that while including the $2 p 1 h$ and $1 p 2 h$ spaces when diagonalizing the EOM-CC effective Hamiltonian results in an effective screening of the principal poles of the Green's function [54], the effective Hamiltonian matrix elements do not themselves properly contain screened interactions. To screen the particle/hole interactions in the trion thus requires including the $3 p 2 h$ and $2 p 3 h$ spaces when diagonalizing the EOM-CC effective Hamiltonian, which incurs a greater expense.

In summary, we have found that EOM-CCSD approximation can be applied in practice to access the electronic band gap and band structure of monolayer $2 H$-TMDs. We analyzed the form of the convergence to the thermodynamic limit in two dimensions, which is the primary source of error, similarly to that seen in 3D semiconductors [53]. The size of the band 
gaps is in the upper range of those seen in the spread of DFT$G W$ gaps obtained for monolayer $2 H$-TMDs, bounding the effect of remaining many-body correlations on this quantity. Some of the differences between our computed electronic band structure for monolayer $2 \mathrm{H}-\mathrm{MoS}_{2}$ and experimental and earlier theoretical results arise from the neglect of spin-orbit coupling in our Hamiltonian, and thus we estimated spin-orbit coupling effects in a hybrid manner. The CCSD approach is able to qualitatively predict the existence of three-particle excited states, although quantitative energies will require the inclusion of more states when diagonalizing the effective Hamiltonian. Our results illustrate the potential for many- body coupled cluster methods to provide a deeper understanding of the electronic structure of novel two-dimensional materials.

A.P. thanks Swiss NSF for the support provided through the Early Postdoc Mobility program (Project No. P2ELP2_175281). G.K.-L.C. was supported by the US Department of Energy, Office of Science via Grant No. DESC0018140 for this work. Secondary support for PYSCF software infrastructure was provided by the U.S. National Science Foundation via Grant No. 1657286. We thank Q. Sun and J. McClain for valuable discussions.
[1] K. S. Novoselov, D. Jiang, F. Schedin, T. J. Booth, V. V. Khotkevich, S. V. Morozov, and A. K. Geim, Proc. Natl. Acad. Sci. USA 102, 10451 (2005).

[2] S. Z. Butler, S. M. Hollen, L. Cao, Y. Cui, J. A. Gupta, H. R. Gutiérrez, T. F. Heinz, S. S. Hong, J. Huang, A. F. Ismach, E. Johnston-Halperin, M. Kuno, V. V. Plashnitsa, R. D. Robinson, R. S. Ruoff, S. Salahuddin, J. Shan, L. Shi, M. G. Spencer, M. Terrones et al., ACS Nano 7, 2898 (2013).

[3] A. B. Kaul, J. Mater. Res. 29, 348 (2014).

[4] A. Splendiani, L. Sun, Y. Zhang, T. Li, J. Kim, C.-Y. Chim, G. Galli, and F. Wang, Nano Lett. 10, 1271 (2010).

[5] Y.-H. Lee, X.-Q. Zhang, W. Zhang, M.-T. Chang, C.-T. Lin, K.-D. Chang, Y.-C. Yu, J. T.-W. Wang, C.-S. Chang, L.-J. Li, and T.-W. Lin, Adv. Mater. 24, 2320 (2012).

[6] Y. Zhang, T.-R. Chang, B. Zhou, Y.-T. Cui, H. Yan, Z. Liu, F. Schmitt, J. Lee, R. Moore, Y. Chen, H. Lin, H.-T. Jeng, S.-K. Mo, Z. Hussain, A. Bansil, and Z.-X. Shen, Nat. Nanotechnol. 9, 111 (2014).

[7] K. F. Mak, C. Lee, J. Hone, J. Shan, and T. F. Heinz, Phys. Rev. Lett. 105, 136805 (2010).

[8] C. L. Kane and E. J. Mele, Phys. Rev. Lett. 95, 226801 (2005).

[9] X. Qian, J. Liu, L. Fu, and J. Li, Science 346, 1344 (2014).

[10] N. Samarth, Nature (London) 546, 216 (2017).

[11] M. Gibertini, M. Koperski, A. F. Morpurgo, and K. S. Novoselov, Nat. Nanotechnol. 14, 408 (2019).

[12] V. Fatemi, S. Wu, Y. Cao, L. Bretheau, Q. D. Gibson, K. Watanabe, T. Taniguchi, R. J. Cava, and P. Jarillo-Herrero, Science 362, 926 (2018).

[13] C. Xu and L. Balents, Phys. Rev. Lett. 121, 087001 (2018).

[14] D. Xiao, G.-B. Liu, W. Feng, X. Xu, and W. Yao, Phys. Rev. Lett. 108, 196802 (2012).

[15] X. Xi, Z. Wang, W. Zhao, J.-H. Park, K. T. Law, H. Berger, L. Forró, J. Shan, and K. F. Mak, Nat. Phys. 12, 139 (2016).

[16] B. Radisavljevic, M. B. Whitwick, and A. Kis, ACS Nano 5, 9934 (2011).

[17] B. Radisavljevic, A. Radenovic, J. Brivio, V. Giacometti, and A. Kis, Nat. Nanotechnol. 6, 147 (2011).

[18] Q. H. Wang, K. Kalantar-Zadeh, A. Kis, J. N. Coleman, and M. S. Strano, Nat. Nanotechnol. 7, 699 (2012).

[19] B. W. H. Baugher, H. O. H. Churchill, Y. Yang, and P. JarilloHerrero, Nano Lett. 13, 4212 (2013).

[20] X. Duan, C. Wang, A. Pan, R. Yu, and X. Duan, Chem. Soc. Rev. 44, 8859 (2015).
[21] K. F. Mak, K. He, J. Shan, and T. F. Heinz, Nat. Nanotechnol. 7, 494 (2012).

[22] M. Bernardi, M. Palummo, and J. C. Grossman, Nano Lett. 13, 3664 (2013).

[23] M. M. Ugeda, A. J. Bradley, S.-F. Shi, F. H. da Jornada, Y. Zhang, D. Y. Qiu, W. Ruan, S.-K. Mo, Z. Hussain, Z.-X. Shen, F. Wang, S. G. Louie, and M. F. Crommie, Nat. Mater. 13, 1091 (2014).

[24] M. M. Ugeda, A. Pulkin, S. Tang, H. Ryu, Q. Wu, Y. Zhang, D. Wong, Z. Pedramrazi, A. Martín-Recio, Y. Chen, F. Wang, Z.-X. Shen, S.-K. Mo, O. V. Yazyev, and M. F. Crommie, Nat. Commun. 9, 3401 (2018).

[25] K. F. Mak, K. He, C. Lee, G. H. Lee, J. Hone, T. F. Heinz, and J. Shan, Nat. Mater. 12, 207 (2013).

[26] W. Jin, P.-C. Yeh, N. Zaki, D. Zhang, J. T. Sadowski, A. AlMahboob, A. M. van der Zande, D. A. Chenet, J. I. Dadap, I. P. Herman, P. Sutter, J. Hone, and R. M. Osgood, Phys. Rev. Lett. 111, 106801 (2013).

[27] N. Alidoust, G. Bian, S.-Y. Xu, R. Sankar, M. Neupane, C. Liu, I. Belopolski, D.-X. Qu, J. D. Denlinger, F.-C. Chou, and M. Z. Hasan, Nat. Commun. 5, 4673 (2014).

[28] W. Jin, P.-C. Yeh, N. Zaki, D. Zhang, J. T. Liou, J. T. Sadowski, A. Barinov, M. Yablonskikh, J. I. Dadap, P. Sutter, I. P. Herman, and R. M. Osgood, Phys. Rev. B 91, 121409(R) (2015).

[29] P. Vancsó, G. Z. Magda, J. Pet, J.-Y. Noh, Y.-S. Kim, C. Hwang, L. P. Biró, and L. Tapasztó, Sci. Rep. 6, 29726 (2016).

[30] H. M. Hill, A. F. Rigosi, K. T. Rim, G. W. Flynn, and T. F. Heinz, Nano Lett. 16, 4831 (2016).

[31] T. Li and G. Galli, J. Phys. Chem. C 111, 16192 (2007).

[32] Z. Y. Zhu, Y. C. Cheng, and U. Schwingenschlögl, Phys. Rev. B 84, 153402 (2011).

[33] J. K. Ellis, M. J. Lucero, and G. E. Scuseria, Appl. Phys. Lett. 99, 261908 (2011).

[34] H.-P. Komsa and A. V. Krasheninnikov, Phys. Rev. B 86, 241201(R) (2012).

[35] T. Cheiwchanchamnangij and W. R. L. Lambrecht, Phys. Rev. B 85, 205302 (2012).

[36] P. Johari and V. B. Shenoy, ACS Nano 6, 5449 (2012).

[37] A. Kumar and P. K. Ahluwalia, Eur. Phys. J. B 85, 1 (2012).

[38] A. Ramasubramaniam, Phys. Rev. B 86, 115409 (2012).

[39] D. Y. Qiu, F. H. da Jornada, and S. G. Louie, Phys. Rev. Lett. 111, 216805 (2013). 
[40] H. Shi, H. Pan, Y.-W. Zhang, and B. I. Yakobson, Phys. Rev. B 87, 155304 (2013).

[41] F. Zahid, L. Liu, Y. Zhu, J. Wang, and H. Guo, AIP Adv. 3, 052111 (2013).

[42] F. A. Rasmussen and K. S. Thygesen, J. Phys. Chem. C 119, 13169 (2015).

[43] D. Y. Qiu, F. H. da Jornada, and S. G. Louie, Phys. Rev. B 93, 235435 (2016).

[44] M. Drüppel, T. Deilmann, J. Noky, P. Marauhn, P. Krüger, and M. Rohlfing, Phys. Rev. B 98, 155433 (2018).

[45] S. Barja, S. Refaely-Abramson, B. Schuler, D. Y. Qiu, A. Pulkin, S. Wickenburg, H. Ryu, M. M. Ugeda, C. Kastl, C. Chen, C. Hwang, A. Schwartzberg, S. Aloni, S.-K. Mo, D. F. Ogletree, M. F. Crommie, O. V. Yazyev, S. G. Louie, J. B. Neaton, and A. Weber-Bargioni, Nat. Commun. 10, 3382 (2019).

[46] I. C. Gerber and X. Marie, Phys. Rev. B 98, 245126 (2018).

[47] Y. J. Zheng, Y. Chen, Y. L. Huang, P. K. Gogoi, M.-Y. Li, L.-J. Li, P. E. Trevisanutto, Q. Wang, S. J. Pennycook, A. T. S. Wee, and S. Y. Quek, ACS Nano 13, 6050 (2019).

[48] S. Hirata, I. Grabowski, M. Tobita, and R. J. Bartlett, Chem. Phys. Lett. 345, 475 (2001).

[49] S. Hirata, R. Podeszwa, M. Tobita, and R. J. Bartlett, J. Chem. Phys. 120, 2581 (2004).

[50] H. Katagiri, J. Chem. Phys. 122, 224901 (2005).

[51] G. H. Booth, A. Grüneis, G. Kresse, and A. Alavi, Nature (London) 493, 365 (2013).

[52] J. Yang, W. Hu, D. Usvyat, D. Matthews, M. Schütz, and G. K.L. Chan, Science 345, 640 (2014).

[53] J. McClain, Q. Sun, G. K.-L. Chan, and T. C. Berkelbach, J. Chem. Theory Comput. 13, 1209 (2017).

[54] M. F. Lange and T. C. Berkelbach, J. Chem. Theory Comput. 14, 4224 (2018).

[55] J. McClain, J. Lischner, T. Watson, D. A. Matthews, E. Ronca, S. G. Louie, T. C. Berkelbach, and G. K.-L. Chan, Phys. Rev. B 93, 235139 (2016).

[56] N. Godbout, D. R. Salahub, J. Andzelm, and E. Wimmer, Can. J. Chem. 70, 560 (1992).

[57] F. Weigend and R. Ahlrichs, Phys. Chem. Chem. Phys. 7, 3297 (2005).

[58] Q. Sun, T. C. Berkelbach, N. S. Blunt, G. H. Booth, S. Guo, Z. Li, J. Liu, J. D. McClain, E. R. Sayfutyarova, S. Sharma, S. Wouters, and G. K.-L. Chan, Wiley Interdiscip. Rev.: Comput. Mol. Sci. 8, e1340 (2018).
[59] Q. Sun, T. C. Berkelbach, J. D. McClain, and G. K.-L. Chan, J. Chem. Phys. 147, 164119 (2017).

[60] See Supplemental Material at http://link.aps.org/supplemental/ 10.1103/PhysRevB.101.241113 for details of coupled cluster simulations.

[61] F. Gygi and A. Baldereschi, Phys. Rev. B 34, 4405 (1986).

[62] M. S. Hybertsen and S. G. Louie, Phys. Rev. B 34, 5390 (1986).

[63] J. Paier, R. Hirschl, M. Marsman, and G. Kresse, J. Chem. Phys. 122, 234102 (2005).

[64] M. Shishkin and G. Kresse, Phys. Rev. B 74, 035101 (2006).

[65] K. Liao and A. Grüneis, J. Chem. Phys. 145, 141102 (2016).

[66] T. Gruber, K. Liao, T. Tsatsoulis, F. Hummel, and A. Grüneis, Phys. Rev. X 8, 021043 (2018).

[67] Y. Yang, V. Gorelov, C. Pierleoni, D. M. Ceperley, and M. Holzmann, Phys. Rev. B 101, 085115 (2020).

[68] R. Sundararaman and T. A. Arias, Phys. Rev. B 87, 165122 (2013).

[69] D. Liu, Y. Guo, L. Fang, and J. Robertson, Appl. Phys. Lett. 103, 183113 (2013).

[70] H. Peelaers and C. G. Van de Walle, Phys. Rev. B 86, 241401(R) (2012).

[71] H. J. Conley, B. Wang, J. I. Ziegler, R. F. Haglund, S. T. Pantelides, and K. I. Bolotin, Nano Lett. 13, 3626 (2013).

[72] K. He, C. Poole, K. F. Mak, and J. Shan, Nano Lett. 13, 2931 (2013).

[73] F. Hüser, T. Olsen, and K. S. Thygesen, Phys. Rev. B 88, 245309 (2013).

[74] J. Xi, T. Zhao, D. Wang, and Z. Shuai, J. Phys. Chem. Lett. 5, 285 (2014).

[75] J. Chang, L. F. Register, and S. K. Banerjee, J. Appl. Phys. 115, 084506 (2014).

[76] D. Wickramaratne, F. Zahid, and R. K. Lake, J. Chem. Phys. 140, 124710 (2014).

[77] J. S. Ross, S. Wu, H. Yu, N. J. Ghimire, A. M. Jones, G. Aivazian, J. Yan, D. G. Mandrus, D. Xiao, W. Yao, and X. Xu, Nat. Commun. 4, 1474 (2013).

[78] C. Zhang, H. Wang, W. Chan, C. Manolatou, and F. Rana, Phys. Rev. B 89, 205436 (2014).

[79] J. W. Christopher, B. B. Goldberg, and A. K. Swan, Sci. Rep. 7, 14062 (2017).

[80] A. P. S. Gaur, A. M. Rivera, S. P. Dash, S. Dey, R. S. Katiyar, and S. Sahoo, Appl. Phys. Lett. 115, 173103 (2019).

[81] Z. Li, T. Wang, Z. Lu, M. Khatoniar, Z. Lian, Y. Meng, M. Blei, T. Taniguchi, K. Watanabe, S. A. McGill, S. Tongay, V. M. Menon, D. Smirnov, and S.-F. Shi, Nano Lett. 19, 6886 (2019). 